International Journal of Applied Mathematics

Volume 31 No. $3 \quad 2018,333-348$

ISSN: 1311-1728 (printed version); ISSN: 1314-8060 (on-line version)

doi: http://dx.doi.org/10.12732/ijam.v31i3.3

\title{
EXISTENCE AND UNIQUENESS THEOREMS \\ FOR FRACTIONAL VOLTERRA-FREDHOLM INTEGRO-DIFFERENTIAL EQUATIONS
}

\author{
Ahmed A. Hamoud ${ }^{1}$, Kirtiwant P. Ghadle ${ }^{2}$, \\ M.Sh. Bani Issa ${ }^{3}$, Giniswamy ${ }^{4}$ \\ ${ }^{1,2}$ Department of Mathematics \\ Dr. Babasaheb Ambedkar Marathwada University \\ Aurangabad, 431004, INDIA \\ ${ }^{3,4}$ Department of Mathematics \\ P.E.T. Research Foundation \\ Mandya, University of Mysore, 570401, INDIA
}

\begin{abstract}
In this article, the homotopy perturbation method has been successfully applied to find the approximate solution of a Caputo fractional Volterra-Fredholm integro-differential equation. The reliability of the method and reduction in the size of the computational work give this method a wider applicability. Also, the behavior of the solution can be formally determined by the analytical approximate. Moreover, we proved the existence and uniqueness results of the solution. Finally, an example is included to demonstrate the validity and applicability of the proposed technique.
\end{abstract}

AMS Subject Classification: 26A33, 35C10, 65H20

Key Words: homotopy perturbation method, Caputo fractional VolterraFredholm integro-differential equation, existence and uniqueness results

\section{Introduction}

In the recent years, numerous papers have been concentrating on the development of analytical and numerical methods for fractional order integro-differential equations. In this paper, we consider a Caputo fractional Volterra-Fredholm

$\begin{array}{ll}\text { Received: February 9, } 2018 & \text { (c) } 2018 \text { Academic Publications }\end{array}$

${ }^{\S}$ Correspondence author 
integro-differential equation of the form:

$$
\begin{aligned}
{ }^{c} D^{\alpha} u(x)= & a(x) u(x)+g(x)+\int_{0}^{x} K_{1}(x, t) F_{1}(u(t)) d t \\
& +\int_{0}^{1} K_{2}(x, t) F_{2}(u(t)) d t
\end{aligned}
$$

with the initial condition

$$
y^{(i)}(0)=\delta_{i}, \quad i=0,1,2, \cdots, n-1,
$$

where ${ }^{c} D^{\alpha}$ is the Caputo fractional derivative, $n-1<\alpha \leq n$ and $n \in \mathbb{N}$, $u: J \longrightarrow \mathbb{R}$, where $J=[0,1]$ is the continuous function which has to be determined, $g: J \longrightarrow \mathbb{R}$ and $K_{i}: J \times J \longrightarrow \mathbb{R}$, are continuous functions. $F_{i}: \mathbb{R} \longrightarrow \mathbb{R}, \quad i=1,2$ are Lipschitz continuous functions.

An application of the fractional order derivatives was first given in 1823 by Abel [1] who applied the fractional calculus in the solution of an integral equation that arises in the formulation of the Tautochrone problem. The fractional integro-differential equations have attracted much more interest of mathematicians and physicists which provides an efficiency for the description of many practical dynamical arising in engineering and scientific disciplines such as, physics, biology, electrochemistry, chemistry, economics, electromagnetic, control theory and viscoelasticity $[2,4,5,6,7,8,16,17,20]$. In the recent years, many authors focus on the development of numerical and analytical techniques for fractional integro-differential equations. For instance, we can mention the following works. Al-Samadi and Gumah [3] applied the homotopy analysis method for fractional SEIR epidemic model, Zurigat et al. [22] applied HAM for system of fractional integro-differential equations, Yang and Hou [20] applied the Laplace decomposition method to solve the fractional integro-differential equations, Mittal and Nigam [17] applied the Adomian decomposition method to approximate solutions for fractional integro-differential equations, and $\mathrm{Ma}$ and Huang [16] applied hybrid collocation method to study integro-differential equations of fractional order.

The main objective of the present paper is to study the behavior of the solution that can be formally determined by homotopy perturbation method. Moreover, we prove existence and uniqueness results.

The rest of the paper is organized as follows: In Section 2, some preliminaries and basic definitions related to fractional calculus are recalled. In Section 3, a homotopy perturbation method is constructed for solving Caputo fractional Volterra-Fredholm integro-differential equations. In Section 4, the existence and uniqueness of the solution have been proved. In Section 5, the analytical 
example is presented to illustrate the accuracy of this method. Finally, we will give a report on our paper and a brief conclusion are given in Section 6 .

\section{Preliminaries}

As tools of mathematical analysis, the definitions of fractional derivative and fractional integration are provided by several different approaches. The most frequently used definitions of the operators of fractional calculus involves the Riemann-Liouville fractional derivative and the Caputo derivative, see for example [19, 18, 14, 21].

Definition 1. The Riemann-Liouville fractional integral of order $\alpha>0$ of a function $f$ is defined as

$$
\begin{aligned}
J^{\alpha} f(x) & =\frac{1}{\Gamma(\alpha)} \int_{0}^{x}(x-t)^{\alpha-1} f(t) d t, \quad x>0, \quad \alpha \in \mathbb{R}^{+}, \\
J^{0} f(x) & =f(x),
\end{aligned}
$$

where $\mathbb{R}^{+}$is the set of positive real numbers.

Definition 2. The fractional derivative of $f(x)$ in the Caputo sense is defined by

$$
\begin{aligned}
& { }^{c} D_{x}^{\alpha} f(x)=J^{m-\alpha} D^{m} f(x) \\
& = \begin{cases}\frac{1}{\Gamma(m-\alpha)} \int_{0}^{x}(x-t)^{m-\alpha-1} \frac{d^{m} f(t)}{d t^{m}} d t, & m-1<\alpha<m, \\
\frac{d^{m} f(x)}{d x^{m}}, & \alpha=m, \quad m \in N,\end{cases}
\end{aligned}
$$

where the parameter $\alpha$ is the order of the derivative and is allowed to be real or even complex, with $\Re \alpha>0$. In this paper, only real and positive $\alpha$ will be considered.

The following properties are well known in fractional calculus:

1. $J^{\alpha} J^{v} f=J^{\alpha+v} f, \quad \alpha, v>0$,

2. $J^{\alpha} x^{\beta}=\frac{\Gamma(\beta+1)}{\Gamma(\beta+\alpha+1)} x^{\beta+\alpha}$,

3. $J^{\alpha}{ }^{c} D^{\alpha} f(x)=f(x)-\sum_{k=0}^{m-1} f^{(k)}\left(0^{+}\right) \frac{x^{k}}{k !}, \quad x>0, m-1<\alpha \leq m$. 
Definition 3. The Riemann-Liouville fractional derivative of order $\alpha>0$ is defined as

$$
D^{\alpha} f(x)=D^{m} J^{m-\alpha} f(x), \quad m-1<\alpha \leq m, \quad m \in \mathbb{N} .
$$

Theorem 4. ([21], Banach contraction principle) Let $(X, d)$ be a complete metric space, then each contraction mapping $T: X \longrightarrow X$ has a unique fixed point $x$ of $T$ in $X$, i.e. $T x=x$.

Theorem 5. ([13], Schauder's fixed point theorem) Let $X$ be a Banach space and let $A$ a convex, closed subset of $X$. If $T: A \longrightarrow A$ be the map such that the set $\{T u: u \in A\}$ is relatively compact in $X$ (or $T$ is continuous and completely continuous). Then $T$ has at least one fixed point $u^{*} \in A: T u^{*}=u^{*}$.

\section{Homotopy Perturbation Method (HPM)}

The homotopy perturbation method first proposed by He [10, 11, 12]. To illustrate the basic idea of this method, we consider the following nonlinear differential equation

$$
A(u)-f(r)=0, \quad r \in \Omega,
$$

under the boundary conditions

$$
B\left(u, \frac{\partial u}{\partial n}\right)=0, \quad r \in \Gamma,
$$

where $A$ is a general differential operator, $B$ is a boundary operator, $f(r)$ is a known analytic function, $\Gamma$ is the boundary of the domain $\Omega$.

In general, the operator $A$ can be divided into two parts $L$ and $N$, where $L$ is linear, while $N$ is nonlinear. Eq. (6) therefore can be rewritten as follows $[9]$ :

$$
L(u)+N(u)-f(r)=0 .
$$

By the homotopy technique (Liao 1992, 1997, [15]), we construct a homotopy $v(r, p): \Omega \times[0,1] \longrightarrow \mathbb{R}$ which satisfies

$$
H(v, p)=(1-p)\left[L(v)-L\left(u_{0}\right)\right]+p[A(v)-f(r)]=0, p \in[0,1]
$$

or

$$
\left.H(v, p)=L(v)-L\left(u_{0}\right)+p L\left(u_{0}\right)\right]+p[N(v)-f(r)]=0,
$$


where $p \in[0,1]$ is an embedding parameter, $u_{0}$ is an initial approximation of Eq.(6) which satisfies the boundary conditions. From Eqs.(9), (10) we have

$$
\begin{aligned}
& H(v, 0)=L(v)-L\left(u_{0}\right)=0, \\
& H(v, 1)=A(v)-f(r)=0 .
\end{aligned}
$$

The changing in the process of $p$ from zero to unity is just that of $v(r, p)$ from $u_{0}(r)$ to $u(r)$. In topology this is called deformation and $L(v)-L\left(u_{0}\right)$, and $A(v)-f(r)$ are called homotopic. Now, assume that the solution of Eqs. (9), (10) can be expressed as

$$
v=v_{0}+p v_{1}+p^{2} v_{2}+\cdots .
$$

The approximate solution of Eq.(6) can be obtained by setting $p=1$, namely

$$
u=\lim _{p \rightarrow 1} v=v_{0}+v_{1}+v_{2}+\cdots \text {. }
$$

\subsection{Homotopy Perturbation Method Applied to Caputo Fractional Volterra-Fredholm Integro-Differential Equation}

We consider Caputo fractional Volterra-Fredholm integro-differential equation given by (1), with the initial condition (2).

We can define

$$
\begin{aligned}
& (1-P)^{c} D^{\alpha} u(x)+P\left[{ }^{c} D^{\alpha} u(x)-a(x) u(x)-g(x)\right. \\
& \left.-\int_{0}^{x} K_{1}(x, t) F_{1}(u(t)) d t-\int_{0}^{1} K_{2}(x, t) F_{2}(u(t)) d t\right]=0 .
\end{aligned}
$$

In view of the basic assumption of HPM, solution of (1) can be expressed as a power series in $P$ :

$$
u(x)={ }^{c} D^{\alpha} u_{0}(x)+P^{c} D^{\alpha} u_{1}(x)+P^{2 c} D^{\alpha} u_{2}(x)+P^{3 c} D^{\alpha} u_{3}(x)+\cdots .
$$

If we put $P \longrightarrow 1$ in (16), we get the approximate solution of (1):

$$
u(x)={ }^{c} D^{\alpha} u_{0}(x)+{ }^{c} D^{\alpha} u_{1}(x)+{ }^{c} D^{\alpha} u_{2}(x)+{ }^{c} D^{\alpha} u_{3}(x)+\cdots .
$$

Now, we substitute (16) into (15), then equating the terms with identical power of $P$, we obtain the following series of linear equations:

$$
\begin{aligned}
& P^{0}:{ }^{c} D^{\alpha} u_{0}(x)=0 \\
& P^{1}:{ }^{c} D^{\alpha} u_{1}(x)=g(x)+a(x) u_{0}(x)+\int_{0}^{x} K_{1}(x, t) F_{1}\left(u_{0}(t)\right) d t
\end{aligned}
$$




$$
\begin{aligned}
& +\int_{0}^{1} K_{2}(x, t) F_{2}\left(u_{0}(t)\right) d t, \\
P^{2}:{ }^{c} D^{\alpha} u_{2}(x)= & a(x) u_{1}(x)+\int_{0}^{x} K_{1}(x, t) F_{1}\left(u_{1}(t)\right) d t \\
& +\int_{0}^{1} K_{2}(x, t) F_{2}\left(u_{1}(t)\right) d t, \\
P^{3}:{ }^{c} D^{\alpha} u_{3}(x)= & a(x) u_{2}(x)+\int_{0}^{x} K_{1}(x, t) F_{1}\left(u_{2}(t)\right) d t \\
& +\int_{0}^{1} K_{2}(x, t) F_{2}\left(u_{2}(t)\right) d t,
\end{aligned}
$$

\section{The Main Results}

In this section, we shall give an existence and uniqueness results of Eq.(1), with the initial condition (2) and prove these. Before starting and proving the main results, we introduce the following hypotheses:

(A1) There exist two constants $L_{F_{1}}, L_{F_{2}}>0$ such that, for any $u_{1}, u_{2} \in$ $C(J, \mathbb{R})$

$$
\left|F_{1}\left(u_{1}(x)\right)-F_{1}\left(u_{2}(x)\right)\right| \leq L_{F_{1}}\left|u_{1}-u_{2}\right|
$$

and

$$
\left|F_{2}\left(u_{1}(x)\right)-F_{2}\left(u_{2}(x)\right)\right| \leq L_{F_{2}}\left|u_{1}-u_{2}\right|
$$

(A2) There exist two functions $K_{1}^{*}, K_{2}^{*} \in C\left(D, \mathbb{R}^{+}\right)$, the set of all positive function continuous on $D=\{(x, t) \in \mathbb{R} \times \mathbb{R}: 0 \leq t \leq x \leq 1\}$ such that

$$
K_{1}^{*}=\sup _{x, t \in[0,1]} \int_{0}^{x}\left|K_{1}(x, t)\right| d t<\infty, K_{2}^{*}=\sup _{x, t \in[0,1]} \int_{0}^{x}\left|K_{2}(x, t)\right| d t<\infty .
$$

(A3) The two functions $a, g: J \rightarrow \mathbb{R}$ are continuous.

Lemma 6. If $u_{0}(x) \in C(J, \mathbb{R})$, then $u(x) \in C\left(J, \mathbb{R}^{+}\right)$is a solution of the problem (1) - (2) iff $u$ is satisfying

$$
u(x)=u_{0}+\frac{1}{\Gamma(\alpha)} \int_{0}^{x}(x-s)^{\alpha-1} a(s) u(s) d s
$$




$$
\begin{aligned}
& +\frac{1}{\Gamma(\alpha)} \int_{0}^{x}(x-s)^{\alpha-1} g(s) d s+\frac{1}{\Gamma(\alpha)} \int_{0}^{x}(x-s)^{\alpha-1} \\
& \times\left(\int_{0}^{s} K_{1}(s, \tau) F_{1}(u(\tau)) d \tau+\int_{0}^{1} K_{2}(s, \tau) F_{2}(u(\tau)) d \tau\right) d s
\end{aligned}
$$

for $x \in J$, and $u_{0}=\sum_{k=0}^{n-1} u^{k}\left(0^{+}\right) \frac{x^{k}}{k !}$.

Our first result is based on the Banach contraction principle.

Theorem 7. Assume that (A1), (A2) and (A3) hold. If

$$
\left(\frac{\|a\|_{\infty}+K_{1}^{*} L_{F_{1}}+K_{2}^{*} L_{F_{2}}}{\Gamma(\alpha+1)}\right)<1 .
$$

Then there exists a unique solution $u(x) \in C(J)$ to $(1)-(2)$.

Proof. By Lemma 6, we know that a function $u$ is a solution to (1) - (2) iff $u$ satisfies Eq. (19). Let the operator $T: C(J, \mathbb{R}) \rightarrow C(J, \mathbb{R})$ be defined by

$$
\begin{aligned}
(T u)(x)= & u_{0}+\frac{1}{\Gamma(\alpha)} \int_{0}^{x}(x-s)^{\alpha-1} a(s) u(s) d s \\
& +\frac{1}{\Gamma(\alpha)} \int_{0}^{x}(x-s)^{\alpha-1} g(s) d s+\frac{1}{\Gamma(\alpha)} \int_{0}^{x}(x-s)^{\alpha-1} \\
& \times\left(\int_{0}^{s} K_{1}(s, \tau) F_{1}(u(\tau)) d \tau+\int_{0}^{1} K_{2}(s, \tau) F_{2}(u(\tau)) d \tau\right) d s,
\end{aligned}
$$

we can see that, if $u \in C(J, \mathbb{R})$ is a fixed point of $T$, then $u$ is a solution of (1) $-(2)$.

Now we prove $T$ has a fixed point $u$ in $C(J, \mathbb{R})$. For that, let $u_{1}, u_{2} \in C(J, \mathbb{R})$ and for any $x \in[0,1]$ such that

$$
\begin{aligned}
u_{1}(x)= & u_{0}+\frac{1}{\Gamma(\alpha)} \int_{0}^{x}(x-s)^{\alpha-1} a(s) u_{1}(s) d s \\
& +\frac{1}{\Gamma(\alpha)} \int_{0}^{x}(x-s)^{\alpha-1} g(s) d s+\frac{1}{\Gamma(\alpha)} \int_{0}^{x}(x-s)^{\alpha-1} \\
& \times\left(\int_{0}^{s} K_{1}(s, \tau) F_{1}\left(u_{1}(\tau)\right) d \tau+\int_{0}^{1} K_{2}(s, \tau) F_{2}\left(u_{1}(\tau)\right) d \tau\right) d s,
\end{aligned}
$$

and,

$$
u_{2}(x)=u_{0}+\frac{1}{\Gamma(\alpha)} \int_{0}^{x}(x-s)^{\alpha-1} a(s) u_{2}(s) d s
$$




$$
\begin{aligned}
& +\frac{1}{\Gamma(\alpha)} \int_{0}^{x}(x-s)^{\alpha-1} g(s) d s+\frac{1}{\Gamma(\alpha)} \int_{0}^{x}(x-s)^{\alpha-1} \\
& \times\left(\int_{0}^{s} K_{1}(s, \tau) F_{1}\left(u_{2}(\tau)\right) d \tau+\int_{0}^{1} K_{2}(s, \tau) F_{2}\left(u_{2}(\tau)\right) d \tau\right) d s .
\end{aligned}
$$

Consequently, we get

$$
\begin{aligned}
& \left|\left(T u_{1}\right)(x)-\left(T u_{2}\right)(x)\right| \\
\leq & \frac{1}{\Gamma(\alpha)} \int_{0}^{x}(x-s)^{\alpha-1}|a(s)|\left|u_{1}(s)-u_{2}(s)\right| d s+\frac{1}{\Gamma(\alpha)} \int_{0}^{x} \\
& \times(x-s)^{\alpha-1}\left(\begin{array}{c}
\int_{0}^{s}\left|K_{1}(s, \tau)\right|\left|F_{1}\left(u_{1}(\tau)\right)-F_{1}\left(u_{2}(\tau)\right)\right| d \tau \\
+\int_{0}^{1}\left|K_{2}(s, \tau)\right|\left|F_{2}\left(u_{1}(\tau)\right)-F_{2}\left(u_{2}(\tau)\right)\right| d \tau
\end{array}\right) d s \\
\leq & \left(\frac{\|a\|_{\infty}}{\Gamma(\alpha+1)}+\frac{K_{1}^{*} L_{F_{1}}}{\Gamma(\alpha+1)}+\frac{K_{2}^{*} L_{F_{2}}}{\Gamma(\alpha+1)}\right)\left|u_{1}(x)-u_{2}(x)\right| \\
= & \left(\frac{\|a\|_{\infty}+K_{1}^{*} L_{F_{1}}+K_{2}^{*} L_{F_{2}}}{\Gamma(\alpha+1)}\right)\left|u_{1}(x)-u_{2}(x)\right| .
\end{aligned}
$$

From the inequality (20) we have

$$
\left\|T u_{1}-T u_{2}\right\|_{\infty} \leq\left\|u_{1}-u_{2}\right\|_{\infty} .
$$

This means that $T$ is contraction map. By the Banach contraction principle, we can conclude that $T$ has a unique fixed point $u$ in $C(J, \mathbb{R})$.

Now, we will study the existence result by means of Schauder's fixed point theorem.

Theorem 8. Assume that $F_{1}, F_{2}$ are continuous functions and (A2), (A3) hold, if

$$
\frac{\|a\|_{\infty}}{\Gamma(\alpha+1)}<1 .
$$

Then there exists at least a solution $u(x) \in C(J, \mathbb{R})$ to problem $(1)-(2)$.

Proof. Let the operator $T: C(J, \mathbb{R}) \rightarrow C(J, \mathbb{R})$, be defined as in Theorem 7.

First, we prove that the operator $T$ is completely continuous.

(1) We show that $T$ is continuous.

Let $u_{n}$ be a sequence such that $u_{n} \rightarrow u$ in $C(J, \mathbb{R})$. Then for each $u_{n}, u$ $\in C(J, \mathbb{R})$ and for any $x \in J$ we have

$$
\left|\left(T u_{n}\right)(x)-(T u)(x)\right|
$$




$$
\begin{aligned}
& \leq \frac{1}{\Gamma(\alpha)} \int_{0}^{x}(x-s)^{\alpha-1}|a(s)|\left|u_{n}(s)-u(s)\right| d s+\frac{1}{\Gamma(\alpha)} \\
& \times \int_{0}^{x}(x-s)^{\alpha-1}\left(\begin{array}{c}
\int_{0}^{s}\left|K_{1}(s, \tau)\right|\left|F_{1}\left(u_{n}(\tau)\right)-F_{1}(u(\tau))\right| d \tau \\
+\int_{0}^{1}\left|K_{2}(s, \tau)\right|\left|F_{2}\left(u_{n}(\tau)\right)-F_{2}(u(\tau))\right| d \tau
\end{array}\right) d s \\
& \leq \frac{1}{\Gamma(\alpha)} \int_{0}^{x}(x-s)^{\alpha-1} \sup _{s \in J}|a(s)| \sup _{s \in J}\left|u_{n}(s)-u(s)\right| d s \\
&+\frac{1}{\Gamma(\alpha)} \int_{0}^{x}(x-s)^{\alpha-1} \\
& \times\left(\begin{array}{c}
\quad \sup _{s, \tau \in J} \int_{0}^{\tau}\left|K_{1}(s, \tau)\right| \sup _{\tau \in J}\left|F_{1}\left(u_{n}(\tau)\right)-F_{1}(u(\tau))\right| d \tau \\
\quad \sup _{s, \tau \in J} \int_{0}^{1}\left|K_{2}(s, \tau)\right| \sup _{\tau \in J}\left|F_{2}\left(u_{n}(\tau)\right)-F_{2}(u(\tau))\right| d \tau
\end{array}\right) d s \\
& \leq\|a\|_{\infty}\left\|u_{n}(.)-u(.)\right\|_{\infty} \frac{1}{\Gamma(\alpha)} \int_{0}^{x}(x-s)^{\alpha-1} d s \\
& \quad+K_{1}^{*}\left\|F_{1}\left(u_{n}(.)\right)-F_{1}(u(.))\right\|_{\infty} \frac{1}{\Gamma(\alpha)} \int_{0}^{x}(x-s)^{\alpha-1} d s \\
& \quad+K_{2}^{*}\left\|F_{2}\left(u_{n}(.)\right)-F_{2}(u(.))\right\|_{\infty} \frac{1}{\Gamma(\alpha)} \int_{0}^{x}(x-s)^{\alpha-1} d s .
\end{aligned}
$$

Since $\int_{0}^{x}(x-s)^{\alpha-1} d s$ is bounded, $\lim _{n \rightarrow \infty} u_{n}(x)=u(x)$ and $F_{1}, F_{2}$ are continuous functions, we conclude that $\left\|T u_{n}-T u\right\|_{\infty} \rightarrow 0$ as $n \rightarrow \infty$, thus, $T$ is continuous on $C(J, \mathbb{R})$.

(2) We verify that $T$ maps bounded sets into bounded sets in $C(J, \mathbb{R})$.

Indeed, just we show that for any $\lambda>0$ there exists a positive constant $\ell$ such that for each $u \in \mathbb{B}_{\lambda}=\left\{u \in C(J, \mathbb{R}):\|u\|_{\infty} \leq \lambda\right\}$, one has $\|T u\|_{\infty} \leq \ell$.

Let $\mu_{1}=\sup _{(u) \in J \times[0, \lambda]} F_{1}(u(x))+1$, and $\mu_{2}=\sup _{(u) \in J \times[0, \lambda]} F_{2}(u(x))+1$, and for any $u \in \mathbb{B}_{r}$ and for each $x \in J$, we have

$$
\begin{aligned}
& |(T u)(x)| \\
= & \left|u_{0}\right|+\frac{1}{\Gamma(\alpha)} \int_{0}^{x}(x-s)^{\alpha-1}|a(s)||u(s)| d s \\
& +\frac{1}{\Gamma(\alpha)} \int_{0}^{x}(x-s)^{\alpha-1}|g(s)| d s+\frac{1}{\Gamma(\alpha)} \int_{0}^{x}(x-s)^{\alpha-1} \\
& \times\left(\int_{0}^{s}\left|K_{1}(s, \tau)\right|\left|F_{1}(u(\tau))\right| d \tau+\int_{0}^{1}\left|K_{2}(s, \tau)\right|\left|F_{2}(u(\tau))\right| d \tau\right) d s \\
\leq & \left|u_{0}\right|+\|u\|_{\infty}\|a\|_{\infty} \frac{x^{\alpha}}{\Gamma(\alpha+1)}+\|g\|_{\infty} \frac{x^{\alpha}}{\Gamma(\alpha+1)}+\frac{K_{1}^{*} \mu_{1} x^{\alpha}}{\Gamma(\alpha+1)}
\end{aligned}
$$




$$
\begin{aligned}
& +\frac{K_{2}^{*} \mu_{2} x^{\alpha}}{\Gamma(\alpha+1)} \\
\leq & \left(\left|u_{0}\right|+\frac{\|a\|_{\infty} \lambda+\|g\|_{\infty}+K_{1}^{*} \mu_{1}+K_{2}^{*} \mu_{2}}{\Gamma(\alpha+1)}\right):=\ell .
\end{aligned}
$$

Therefore, $\|T u\| \leq \ell$ for every $u \in \mathbb{B}_{r}$, which implies that $T \mathbb{B}_{r} \subset \mathbb{B}_{\ell}$.

(3) We examine that $T$ maps bounded sets into equicontinuous sets of $C(J, \mathbb{R})$.

Let $\mathbb{B}_{\lambda}$ is defined as in $(\mathbf{2})$ and for each $u \in \mathbb{B}_{\lambda}, x_{1}, x_{2} \in[0,1]$, with $x_{1}<x_{2}$ we have

$$
\begin{aligned}
& \left|(T u)\left(x_{2}\right)-(T u)\left(x_{1}\right)\right| \\
& \leq \frac{1}{\Gamma(\alpha)}\left|\int_{0}^{x_{2}}\left(x_{2}-s\right)^{\alpha-1} a(s) u(s) d s-\int_{0}^{x_{1}}\left(x_{1}-s\right)^{\alpha-1} a(s) u(s) d s\right| \\
& +\frac{1}{\Gamma(\alpha)}\left|\int_{0}^{x_{2}}\left(x_{2}-s\right)^{\alpha-1} g(s) d s-\int_{0}^{x_{1}}\left(x_{1}-s\right)^{\alpha-1} g(s) d s\right|+\frac{1}{\Gamma(\alpha)} \\
& \int_{0}^{x_{2}}\left(x_{2}-s\right)^{\alpha-1}\left(\int_{0}^{s} K_{1}(s, \tau) F_{1}(u(\tau)) d \tau+\int_{0}^{1} K_{2}(s, \tau) F_{2}(u(\tau)) d \tau\right) d s \\
& -\int_{0}^{x_{1}}\left(x_{1}-s\right)^{\alpha-1}\left(\int_{0}^{s} K_{1}(s, \tau) F_{1}(u(\tau)) d \tau+\int_{0}^{1} K_{2}(s, \tau) F_{2}(u(\tau)) d \tau\right) d s \\
& =\frac{1}{\Gamma(\alpha)} \mid \begin{array}{c}
\int_{0}^{x_{2}}\left(x_{2}-s\right)^{\alpha-1} a(s) u(s) d s-\int_{0}^{x_{1}}\left(x_{2}-s\right)^{\alpha-1} a(s) u(s) d s \\
+\int_{0}^{x_{1}}\left(x_{2}-s\right)^{\alpha-1} a(s) u(s) d s-\int_{0}^{x_{1}}\left(x_{1}-s\right)^{\alpha-1} a(s) u(s) d s
\end{array} \\
& +\frac{1}{\Gamma(\alpha)}\left|\begin{array}{c}
\int_{0}^{x_{2}}\left(x_{2}-s\right)^{\alpha-1} g(s) d s-\int_{0}^{x_{1}}\left(x_{2}-s\right)^{\alpha-1} g(s) d s \\
+\int_{0}^{x_{1}}\left(x_{2}-s\right)^{\alpha-1} g(s) d s-\int_{0}^{x_{1}}\left(x_{1}-s\right)^{\alpha-1} g(s) d s
\end{array}\right|+\frac{1}{\Gamma(\alpha)} \\
& \int_{0}^{x_{2}}\left(x_{2}-s\right)^{\alpha-1}\left(\int_{0}^{s} K_{1}(s, \tau) F_{1}(u(\tau)) d \tau+\int_{0}^{1} K_{2}(s, \tau) F_{2}(u(\tau)) d \tau\right) d s \\
& -\int_{0}^{x_{1}}\left(x_{2}-s\right)^{\alpha-1}\left(\int_{0}^{s} K_{1}(s, \tau) F_{1}(u(\tau)) d \tau+\int_{0}^{1} K_{2}(s, \tau) F_{2}(u(\tau)) d \tau\right) d s \\
& +\int_{0}^{x_{1}}\left(x_{2}-s\right)^{\alpha-1}\left(\int_{0}^{s} K_{1}(s, \tau) F_{1}(u(\tau)) d \tau+\int_{0}^{1} K_{2}(s, \tau) F_{2}(u(\tau)) d \tau\right) d s \\
& -\int_{0}^{x_{1}}\left(x_{1}-s\right)^{\alpha-1}\left(\int_{0}^{s} K_{1}(s, \tau) F_{1}(u(\tau)) d \tau+\int_{0}^{1} K_{2}(s, \tau) F_{2}(u(\tau)) d \tau\right) d s .
\end{aligned}
$$

Consequently,

$$
\begin{aligned}
& \left|(T u)\left(x_{2}\right)-(T u)\left(x_{1}\right)\right| \\
\leq & \frac{1}{\Gamma(\alpha)}\left(\begin{array}{c}
\int_{x_{1}}^{x_{2}}\left(x_{2}-s\right)^{\alpha-1}|a(s)||u(s)| d s \\
+\int_{0}^{x_{1}}\left(x_{1}-s\right)^{\alpha-1}-\left(x_{2}-s\right)^{\alpha-1}|a(s)||u(s)| d s
\end{array}\right) \\
+ & \frac{1}{\Gamma(\alpha)}\left(\begin{array}{c}
\int_{x_{1}}^{x_{2}}\left(x_{2}-s\right)^{\alpha-1}|g(s)| d s \\
+\int_{0}^{x_{1}}\left(x_{1}-s\right)^{\alpha-1}-\left(x_{2}-s\right)^{\alpha-1}|g(s)| d s
\end{array}\right)+\frac{1}{\Gamma(\alpha)}
\end{aligned}
$$




$$
\begin{aligned}
& \left(\begin{array}{c}
\int_{x_{1}}^{x_{2}}\left(x_{2}-s\right)^{\alpha-1}\left(\begin{array}{c}
\int_{0}^{s}\left|K_{1}(s, \tau)\right|\left|F_{1}(u(\tau))\right| d \tau \\
+\int_{0}^{1}\left|K_{2}(s, \tau)\right|\left|F_{2}(u(\tau))\right| d \tau
\end{array}\right) d s+\int_{0}^{x_{1}} \\
\left(x_{1}-s\right)^{\alpha-1}-\left(x_{2}-s\right)^{\alpha-1}\left(\begin{array}{c}
\int_{0}^{s}\left|K_{1}(s, \tau)\right|\left|F_{1}(u(\tau))\right| d \tau \\
+\int_{0}^{1}\left|K_{2}(s, \tau)\right|\left|F_{2}(u(\tau))\right| d \tau
\end{array}\right) d s
\end{array}\right) \\
= & I_{1}+I_{2}+I_{3},
\end{aligned}
$$

where:

$$
\begin{aligned}
I_{1}= & \frac{1}{\Gamma(\alpha)}\left(\int_{x_{1}}^{x_{2}}\left(x_{2}-s\right)^{\alpha-1}|a(s)||u(s)| d s\right. \\
& \left.+\int_{0}^{x_{1}}\left(x_{1}-s\right)^{\alpha-1}-\left(x_{2}-s\right)^{\alpha-1}|a(s)||u(s)| d s\right) \\
\leq & \frac{\left(x_{2}-x_{1}\right)^{\alpha}}{\Gamma(\alpha+1)}\|a\|_{\infty} \lambda+\frac{x_{1}^{\alpha}}{\Gamma(\alpha+1)}\|a\|_{\infty} \lambda+\frac{\left(x_{2}-x_{1}\right)^{\alpha}}{\Gamma(\alpha+1)}\|a\|_{\infty} \lambda \\
& -\frac{x_{2}^{\alpha}}{\Gamma(\alpha+1)}\|a\|_{\infty} \lambda \\
= & \frac{\|a\|_{\infty} \lambda}{\Gamma(\alpha+1)}\left(2\left(x_{2}-x_{1}\right)^{\alpha}+\left(x_{1}^{\alpha}-x_{2}^{\alpha}\right)\right) \\
\leq & \frac{\|a\|_{\infty} \lambda}{\Gamma(\alpha+1)} 2\left(x_{2}-x_{1}\right)^{\alpha}
\end{aligned}
$$

$$
\begin{aligned}
I_{2}= & \frac{1}{\Gamma(\alpha)}\left(\int_{x_{1}}^{x_{2}}\left(x_{2}-s\right)^{\alpha-1}|g(s)| d s\right. \\
& \left.+\int_{0}^{x_{1}}\left(x_{1}-s\right)^{\alpha-1}-\left(x_{2}-s\right)^{\alpha-1}|g(s)| d s\right) \\
\leq & \frac{\left(x_{2}-x_{1}\right)^{\alpha}}{\Gamma(\alpha+1)}\|g\|_{\infty}+\frac{x_{1}^{\alpha}}{\Gamma(\alpha+1)}\|g\|_{\infty}+\frac{\left(x_{2}-x_{1}\right)^{\alpha}}{\Gamma(\alpha+1)}\|g\|_{\infty} \\
& -\frac{x_{2}^{\alpha}}{\Gamma(\alpha+1)}\|g\|_{\infty} \\
= & \frac{\|g\|_{\infty}}{\Gamma(\alpha+1)}\left(2\left(x_{2}-x_{1}\right)^{\alpha}+\left(x_{1}^{\alpha}-x_{2}^{\alpha}\right)\right) \\
\leq & \frac{\|g\|_{\infty}}{\Gamma(\alpha+1)} 2\left(x_{2}-x_{1}\right)^{\alpha}
\end{aligned}
$$


and

$$
\begin{aligned}
I_{3} & =\frac{1}{\Gamma(\alpha)}\left\{\begin{array}{c}
\int_{x_{1}}^{x_{2}}\left(x_{2}-s\right)^{\alpha-1}\left(\begin{array}{c}
\int_{0}^{s}\left|K_{1}(s, \tau)\right|\left|F_{1}(u(\tau))\right| d \tau \\
+\int_{0}^{1}\left|K_{2}(s, \tau)\right|\left|F_{2}(u(\tau))\right| d \tau
\end{array}\right) d s \\
+\int_{0}^{x_{1}}\left(x_{1}-s\right)^{\alpha-1}-\left(x_{2}-s\right)^{\alpha-1} \\
\times\left(\begin{array}{c}
\int_{0}^{s}\left|K_{1}(s, \tau)\right|\left|F_{1}(u(\tau))\right| d \tau \\
+\int_{0}^{1}\left|K_{2}(s, \tau)\right|\left|F_{2}(u(\tau))\right| d \tau
\end{array}\right) d s
\end{array}\right\} \\
\leq & \frac{\left(K_{1}^{*} \mu_{1}+K_{2}^{*} \mu_{2}\right)}{\Gamma(\alpha+1)}\left(2\left(x_{2}-x_{1}\right)^{\alpha}+\left(x_{1}^{\alpha}-x_{2}^{\alpha}\right)\right) \\
\leq & \frac{\left(K_{1}^{*} \mu_{1}+K_{2}^{*} \mu_{2}\right)}{\Gamma(\alpha+1)} 2\left(x_{2}-x_{1}\right)^{\alpha},
\end{aligned}
$$

and we can conclude the right-hand side of (22), (23) and (24) is independently of $u \in \mathbb{B}_{\lambda}$ and tends to zero as $x_{2}-x_{1} \rightarrow 0$. This leads to $\left|(T u)\left(x_{2}\right)-(T u)\left(x_{1}\right)\right| \rightarrow$ 0 as $x_{2} \rightarrow x_{1}$. i.e. the set $\left\{T \mathbb{B}_{\lambda}\right\}$ is equicontinuous.

From $I_{1}$ to $I_{3}$ together with the Arzela-Ascoli theorem, we can conclude that $T: C(J, \mathbb{R}) \rightarrow C(J, \mathbb{R})$ is completely continuous. Finally, we need to investigate that there exists a closed convex bounded subset $\mathbb{B}_{\widetilde{\lambda}}=\left\{u \in C(J, \mathbb{R}):\|u\|_{\infty} \leq\right.$ $\widetilde{\lambda}$ s such that $T \mathbb{B}_{\tilde{\lambda}} \subseteq \mathbb{B}_{\tilde{\lambda}}$. For each positive integer $\widetilde{\lambda}$, then $\mathbb{B}_{\tilde{\lambda}}$ is clearly closed, convex and bounded of $C(J, \mathbb{R})$. We claim that there exists a positive integer $\underset{\widetilde{\lambda}}{\epsilon}$ such that $T \mathbb{B}_{\epsilon} \subseteq \mathbb{B}_{\epsilon}$. If this property is false, then for every positive integer $\widetilde{\lambda}$, there exists $u_{\tilde{\lambda}} \in \mathbb{B}_{\tilde{\lambda}}$ such that $\left(T u_{\tilde{\lambda}}\right) \notin T \mathbb{B}_{\tilde{\lambda}}$, i.e. $\left\|T u_{\tilde{\lambda}}(t)\right\|_{\infty}>\widetilde{\lambda}$ for some $x_{\tilde{\lambda}} \in J$ where $x_{\tilde{\lambda}}$ denotes $x$ depending on $\tilde{\lambda}$. But by using the previous hypotheses we have:

$$
\begin{aligned}
\leq & \left|u_{0}\right|+\|u\|_{\infty}\|a\|_{\infty} \frac{t^{\alpha}}{\Gamma(\alpha+1)}+\|g\|_{\infty} \frac{t^{\alpha}}{\Gamma(\alpha+1)}+\frac{K_{1}^{*} \mu_{1} x^{\alpha}}{\Gamma(\alpha+1)} \\
& +\frac{K_{2}^{*} \mu_{2} x^{\alpha}}{\Gamma(\alpha+1)} \\
\leq & \left(\left|u_{0}\right|+\frac{\|a\|_{\infty} \lambda+\|g\|_{\infty}+K_{1}^{*} \mu_{1}+K_{2}^{*} \mu_{2}}{\Gamma(\alpha+1)}\right) \\
\widetilde{\lambda}< & \left\|T u_{\tilde{\lambda}}\right\|_{\infty} \\
= & \sup _{x \in J}\left|\left(T u_{\tilde{\lambda}}\right)(x)\right| \\
\leq & \sup _{x \in J}\left\{\begin{array}{c}
\left|u_{0}\right|+\left|\frac{1}{\Gamma(\alpha)} \int_{0}^{x}(x-s)^{\alpha-1} a(s)\right| u(s)|d s| \\
+\left|\frac{1}{\Gamma(\alpha)} \int_{0}^{x}(x-s)^{\alpha-1} g(s) d s\right|+\frac{1}{\Gamma(\alpha)} \\
\times \int_{0}^{x}(x-s)^{\alpha-1}\left(\int_{0}^{s}\left|K_{1}(s, \tau)\right|\left|F_{1}(u(\tau))\right| d \tau\right. \\
\left.+\int_{0}^{1}\left|K_{2}(s, \tau)\right|\left|F_{2}(u(\tau))\right| d \tau\right) d s
\end{array}\right\} d s
\end{aligned}
$$




$$
\begin{aligned}
\leq & \sup _{x \in J}\left\{\left|u_{0}\right|+\|u\|_{\infty}\|a\|_{\infty} \frac{x^{\alpha}}{\Gamma(\alpha+1)}+\|g\|_{\infty} \frac{x^{\alpha}}{\Gamma(\alpha+1)}\right. \\
& \left.+\frac{K_{1}^{*} \mu_{1} x^{\alpha}}{\Gamma(\alpha+1)}+\frac{K_{2}^{*} \mu_{2} x^{\alpha}}{\Gamma(\alpha+1)}\right\} \\
\leq & \sup _{x \in J}\left(\left|u_{0}\right|+\frac{\|a\|_{\infty} \tilde{\lambda}+\|g\|_{\infty}+K_{1}^{*} \mu_{1}+K_{2}^{*} \mu_{2}}{\Gamma(\alpha+1)}\right) .
\end{aligned}
$$

Dividing both sides by $\widetilde{\lambda}$ and taking the limit as $\widetilde{\lambda} \rightarrow+\infty$, we obtain

$$
1<\frac{\|a\|_{\infty}}{\Gamma(\alpha+1)}
$$

which contradicts our assumption (21). Hence, for some positive integer $\widetilde{\lambda}$, we must have $T \mathbb{B}_{\tilde{\lambda}} \subseteq \mathbb{B}_{\tilde{\lambda}}$.

An application of Schauder's fixed point theorem shows that there exists at least a fixed point $u$ of $T$ in $C(J, \mathbb{R})$. Then $u$ is the solution to (1) - (2) on $J$, and the proof is completed.

\section{Illustrative Example}

In this section, we present the analytical technique based on HPM to solve Caputo fractional integro-differential equations.

\section{Example 1.}

Let us consider Caputo fractional integro-differential equation:

$$
{ }^{c} D^{\alpha} u(x)=u(x)+\left(1+e^{x}\right) x+3 e^{x}-\int_{0}^{x} u(t) d t, \quad 3<\alpha \leq 4, \quad 0<x<1,
$$

with boundary conditions

$$
\begin{aligned}
& u(0)=1, \quad u^{\prime \prime}(0)=2, \\
& u(1)=1+e, \quad u^{\prime \prime}(1)=3 e .
\end{aligned}
$$

The exact solution of problem (25)-(26) for $\alpha=4$ is

$$
u(x)=1+x e^{x} .
$$


According to the homotopy perturbation method, we construct the following homotopy:

$$
{ }^{c} D^{\alpha} u(x)=P\left(u(x)+\left(1+e^{x}\right) x+3 e^{x}-\int_{0}^{x} u(t) d t\right) .
$$

Substitution of (28) into (18) and then equating the terms with same powers of $P$ yield the following series of linear equations:

$$
\begin{aligned}
& P^{0}:{ }^{c} D^{\alpha} u_{0}(x)=0 \\
& P^{1}:{ }^{c} D^{\alpha} u_{1}(x)=u_{0}(x)+\left(1+e^{x}\right) x+3 e^{x}-\int_{0}^{x} u_{0}(t) d t, \\
& P^{2}:{ }^{c} D^{\alpha} u_{2}(x)=u_{1}(x)-\int_{0}^{x} u_{1}(t) d t, \\
& P^{3}:{ }^{c} D^{\alpha} u_{3}(x)=u_{2}(x)-\int_{0}^{x} u_{2}(t) d t,
\end{aligned}
$$

Applying the operator $J^{\alpha}$ to the above series of linear equations and using initial conditions (26), we get:

$$
\begin{aligned}
u_{0}(x)= & 1, \\
u_{1}(x)= & A x+x^{2}+\frac{B}{6} x^{3}+\frac{4 x^{\alpha}}{\Gamma(\alpha+1)}+\frac{4 x^{\alpha+1}}{\Gamma(\alpha+2)}+\frac{4 x^{\alpha+2}}{\Gamma(\alpha+3)} \\
& +\frac{4 x^{\alpha+3}}{\Gamma(\alpha+4)}+\frac{4 x^{\alpha+4}}{\Gamma(\alpha+5)}, \\
u_{2}(x)= & A \frac{x^{\alpha+1}}{\Gamma(\alpha+2)}+(2-A) \frac{x^{\alpha+2}}{\Gamma(\alpha+3)}+(B-2) \frac{x^{\alpha+3}}{\Gamma(\alpha+4)} \\
& -B \frac{x^{\alpha+4}}{\Gamma(\alpha+5)}+4 \frac{x^{2 \alpha}}{\Gamma(2 \alpha+1)}+\frac{x^{2 \alpha+2}}{\Gamma(2 \alpha+3)}+\frac{x^{2 \alpha+3}}{\Gamma(2 \alpha+4)} \\
& -2 \frac{x^{2 \alpha+4}}{\Gamma(2 \alpha+5)}-4 \frac{x^{2 \alpha+5}}{\Gamma(2 \alpha+6)},
\end{aligned}
$$

where $A$ and $B$ can be determined by imposing boundary conditions.

\section{Conclusions}

The homotopy perturbation method has been applied to derive approximate analytical solutions for fractional Volterra-Fredholm integro-differential equations. Also, we proved the existence and uniqueness of the solution. The illustrative example establishes the precision and efficiency of the proposed technique. 


\section{References}

[1] N. Abel, Solution de quelques problemes a laide dintegrales definites, Christiania Grondahl (Norway) (1881), 16-18.

[2] S. Alkan, V. Hatipoglu, Approximate solutions of Volterra-Fredholm integro-differential equations of fractional order, Tbilisi Mathematical Journal, 10, No 2 (2017), 1-13.

[3] M. AL-Smadi, G. Gumah, On the homotopy analysis method for fractional SEIR epidemic model, Research J. Appl. Sci. Engrg. Technol., 7, No 18 (2014), 3809-3820.

[4] M. Bani Issa, A. Hamoud, K. Ghadle, Giniswamy, Hybrid method for solving nonlinear Volterra-Fredholm integro-differential equations, J. Math. Comput. Sci., 7, No 4 (2017), 625-641.

[5] A. Hamoud, K. Ghadle, The reliable modified of Laplace Adomian decomposition method to solve nonlinear interval Volterra-Fredholm integral equations, Korean J. Math., 25, No 3 (2017), 323-334.

[6] A. Hamoud, K. Ghadle, On the numerical solution of nonlinear VolterraFredholm integral equations by variational iteration method, Int. J. Adv. Sci. Tech. Research, 3 (2016), 45-51.

[7] A. Hamoud, K. Ghadle, The combined modified Laplace with Adomian decomposition method for solving the nonlinear Volterra-Fredholm integrodifferential equations, J. Korean Soc. Ind. Appl. Math., 21 (2017), 17-28.

[8] A. Hamoud, K. Ghadle, Modified Adomian decomposition method for solving fuzzy Volterra-Fredholm integral equations, J. Indian Math. Soc., 85, No (1-2) (2018), 52-69.

[9] A. Hamoud, K. Ghadle, Recent advances on reliable methods for solving Volterra-Fredholm integral and integro-differential equations, Asian J. of Mathematics and Computer Research, 24, No 3 (2018), 128-157.

[10] J.H. He, Homotopy perturbation method: a new nonlinear analytic technique, Appl. Math. Comput., 135 (2003), 73-79.

[11] J.H. He, Homotopy perturbation technique, Computer Methods in Applied Mechanics and Engineering, 178 (1999), 257-262. 
[12] J.H. He, A coupling method of homotopy technique and perturbation technique for nonlinear problems, International Journal of Nonlinear Mechanics, 35 (2000), 37-43.

[13] A. Kilbas, H. Srivastava, J. Trujillo, Theory and Applications of Fractional Differential Equations, North-Holland Math. Stud. Elsevier, Amsterdam, 204 (2006).

[14] V. Lakshmikantham, Theory of fractional functional differential equations, Nonlinear Analysis: Theory, Methods and Appl., 69, No 10 (2008), 33373343.

[15] S. Liao, The Proposed Homotopy Analysis Technique for the Solution of Nonlinear Problems, Ph.D. Thesis, Shanghai Jiao Tong University (1992).

[16] X. Ma, C. Huang, Numerical solution of fractional integro-differential equations by a hybrid collocation method, Appl. Math. Comput., 219, No 12 (2013), 6750-6760.

[17] R. Mittal, R. Nigam, Solution of fractional integro-differential equations by Adomian decomposition method, Int. J. Appl. Math. Mech., 4, No 2 (2008), 87-94.

[18] I. Podlubny, Fractional Differential Equations, Academic Publications, Boston etc. (1999).

[19] S. Samko, A. Kilbas, O. Marichev, Fractional Integrals and Derivatives, Theory and Applications, Gordon and Breach, Yverdon (1993).

[20] C. Yang, J. Hou, Numerical solution of integro-differential equations of fractional order by Laplace decomposition method, WSEAS Trans. Math., 12, No 12 (2013), 1173-1183.

[21] Y. Zhou, Basic Theory of Fractional Differential Equations, Singapore: World Scientific, 6 (2014).

[22] M. Zurigat, S. Momani, A. Alawneh, Homotopy analysis method for systems of fractional integro-differential equations, Neur. Parallel Sci. Comput., 17 (2009), 169-186. 